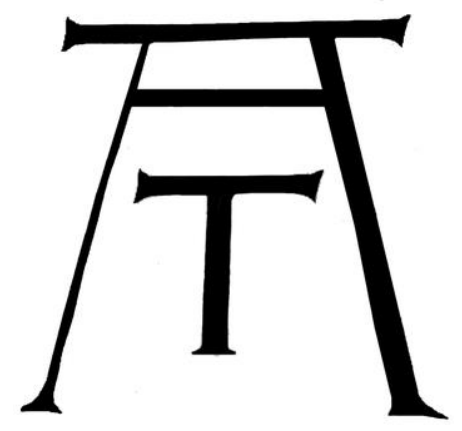

\title{
AsiaTeatro
}

rivista di studi online - ISSN: 2240-4600

www.asiateatro.it

anno 2022, fascicolo n. 1

Giulia Sala

Il Barong turistico: innovazione e tradizione https://doi.org/10.55154/UBUP6899 


\section{Il Barong turistico: innovazione e tradizione}

\section{Giulia Sala}

\section{Sviluppo del turismo nel periodo coloniale e post-coloniale}

Il Barong è una delle forme di teatro danza balinese più rinomate e apprezzate e la sua importanza si è costituita nel tempo affermandosi grazie alla sua valenza esoterica e per l'efficacia scenografica, l'impatto sulla cultura popolare e la sua apparente semplicità strutturale che sottintende invece una profonda conoscenza della tradizione religiosa e spirituale balinese per essere correttamente interpretata. Il legame della danza e di tutta l'arte balinese con la dimensione della religione e del sovrannaturale è indissolubile e si manifesta in ogni rappresentazione. Nato dal dramma rituale del Calonarang e sviluppatosi in seguito come pura forma di intrattenimento, il Barong rappresenta tradizionalmente la lotta tra la figura bestiale benigna del Barong contro Rangda, sotto forma di strega dall'aspetto terrifico e temuta per i suoi poteri di distruzione.

Non è semplice stabilire una netta linea di demarcazione tra la performance e il rituale, nell'ambito del Barong. Lo sviluppo del turismo a Bali ha indubbiamente contribuito a rendere sempre più popolari le sue forme di teatro in tutto il mondo, favorendo maggiormente l'aspetto dell'intrattenimento spettacolare, legato alla musica, alla caratteristica vivacità dei costumi e l'indubbio valore artistico della tradizione delle maschere popolari e dei numerosi stili codificati di danza.

Per l'industria turistica di Bali l'aspetto culturale continua ad essere un ramo ampiamente sviluppato e dopo l'indipendenza dell'Indonesia, dalla metà del '900 sono state attuate una serie di strategie per ristabilire un turismo autoctono e libero dall'influenza olandese, formando guide competenti e aiutando specifiche organizzazioni allo scopo di accrescere e sostenere l'economia locale, dopo la grande difficoltà a seguito delle guerre mondiali. Il turismo ha rappresentato uno dei motori fondamentali per accelerare i cambiamenti sociali, aiutando ad inserire i villaggi balinesi nel periodo del secondo dopoguerra nell'ottica di un sistema capitalistico, anche se il passaggio dal controllo olandese a quello autoctono non ha facilitato il percorso. Bali è stata pubblicizzata anche durante il periodo coloniale mettendo in evidenza il suo retaggio culturale e artistico, e ristabilire in autonomia questo mercato a seguito dell'occupazione 
dei giapponesi ha rappresentato l'obbiettivo principale per favorire uno sviluppo economico.

L'arte ha sempre mantenuto un posto di rilievo nella presentazione esteriore della cultura dell'Indonesia e le danze dei principali festival templari sono state pubblicizzate e riprodotte fin dagli anni '30 su stampe e tele per l'importazione in Occidente, diventando il simbolo dell'isola. Le danzatrici di legong costituirono una delle immagini più gettonate per rappresentare la cultura locale, e insieme a queste la principale figura tradizionale è rimasta la caratteristica maschera di Rangda. L'aspetto così appariscente e aggressivo della figura di Rangda ha fatto in modo che diventasse fin da subito un'icona facilmente riconoscibile per chiunque si interessasse all'Indonesia e a Bali, e ha contribuito alla costruzione consapevole di una raffigurazione "magica" e mistica del luogo, già nel periodo coloniale degli anni ' $30 .{ }^{1}$ I negozi di arte che sorsero allora contribuirono a mantenere l'assetto sociale ed economico dell'isola e ad espandersi anche in ambito commerciale. Nel periodo del dopoguerra, tuttavia, non era semplice ottenere il permesso di visitare Bali.

Questo atteggiamento ambivalente e di chiusura da parte del nuovo governo indipendente di matrice socialista era prevalentemente dovuto a spinte interne in reazione al periodo coloniale. I nazionalisti che avevano combattuto per l'indipendenza si impegnarono a creare le condizioni per accogliere un sempre maggior numero di turisti, in gran parte provenienti dal mondo occidentale, iniziando a progettare alcuni brevi itinerari guidati in cui mostrare piccole parti dell'isola. Nel decennio successivo iniziarono a sorgere le prime forme di intrattenimento artistico pensate e costituite specificatamente per i turisti, che includevano performance di legong, del Barong e di altre danze tradizionali, che accrebbero col tempo la loro nomea nel settore turistico. Gruppi musicali e compagnie di danza furono chiamate ad esibirsi anche per personalità politiche in visita all'isola, nel palazzo presidenziale di Tampaksiring. Uno dei maggiori cambiamenti rispetto al periodo coloniale riguarda infatti la condizione delle giovani danzatrici, inizialmente considerate dagli occidentali collegate alla prostituzione. Una riabilitazione della figura della danzatrice permise di sorpassare questa visione erronea ed equivoca; parte del processo venne facilitato incoraggiando molte figlie di personalità importanti e rispettabili del nuovo governo a praticare la danza, rendendola parte delle

\footnotetext{
${ }^{1}$ Vickers A., 2011, pp. 459-481.
} 
attività culturali raccomandate per l'educazione dell'emergente ceto medio lavoratore balinese.

Gli spettacoli che hanno avuto una maggiore risonanza per il turismo di massa sono, oltre al Barong, anche la danza legong e lo spettacolo delle ombre wayang kulit. L'afflusso turistico e lo sviluppo in direzione della globalizzazione hanno modificato gli equilibri interni al complesso culturale balinese, influenzando le relazioni tra i differenti gruppi culturali ed etnici all'interno della società, favorendo una conoscenza sempre maggiore dei valori culturali esterni. Questo antico retaggio è stato profondamente trasformato prima dal colonialismo e dalla successiva integrazione nel sistema capitalistico mondiale. ${ }^{2}$ La cultura artistica non è rimasta oggetto passivo, ma sono state sviluppate strategie per includere e rivitalizzare nel corso del tempo determinate usanze. Gli spettacoli turistici sono stati perfezionati e adattati all'insegna di una maggiore qualità tecnica, a discapito dell'intrinseco significato religioso. Dal successo dell'industria del turismo in alcuni casi è dipesa la sopravvivenza di determinate tradizioni culturali, che sono state valorizzate in seguito all'incremento dell'interesse esterno.

Non può essere trascurato che nel processo di adattamento per il settore turistico ci sia il rischio di uno slittamento profondo del significato di certe forme di spettacolo. In particolare, c'è una diffusa tendenza a rappresentare in modo manicheo e più definito le entità morali del bene e del male, individuando nello stesso dramma del Barong un protagonista e un antagonista. La figura di Rangda racchiude in sé elementi che rendono impossibile una categorizzazione statica nell'ambito della moralità, rendendo una interpretazione binaria assolutamente inesatta e infedele. È possibile che in alcuni casi l'intrattenimento turistico sia stato orientato ad una progressiva semplificazione e avvicinamento all'interpretazione, operando una modifica sempre più sostanziale del significato originario del dramma.

\section{Scambi culturali tra Bali e altre realtà}

Nel corso degli anni '80 e in quelli successivi molti artisti internazionali e balinesi hanno permesso che la conoscenza delle danze venisse esportata e apprezzata in molti festival artistici di rilievo mondiale, come I Made Bandem, I Wayan Dibia e I Nyoman

\footnotetext{
${ }^{2}$ Wood R.E.,1980, pp. 561-581.
} 
Sedana, che hanno studiato e viaggiato in Australia, Europa e Stati Uniti per poi tornare a stabilirsi come maestri a Bali, contribuendo enormemente a diffondere la cultura balinese all'estero e a favorire un dialogo con le realtà del proprio paese, insieme ad artisti provenienti da tutto il mondo.

Ni Made Wiratini, una danzatrice e maestra di arja, insieme al marito I Wayan Dibia ha esportato questo tipo di danza balinese esibendosi spesso fuori da Bali, ma ha scelto di non partecipare attivamente ai festival all'interno dell'isola, per il rischio di essere esposta a critiche rispetto alla tradizione. Un caso simile ricorda anche Ni Made Pujawati, danzatrice balinese attiva e conosciuta nel Regno Unito che non ha mai lavorato direttamente a Bali. Le danze esportate da Bali e fatte conoscere al grande pubblico europeo e statunitense possono discostarsi dal retaggio di appartenenza e rinnovarsi con più facilità e frequenza rispetto alla realtà da cui provengono. Questo ha permesso una larga diffusione della conoscenza del teatro danza balinese e una maggiore libertà interpretativa per quanto riguarda la trasmissione anche a donne, a persone non autoctone e provenienti da culture estremamente diverse. In alcuni casi certi artisti hanno deliberatamente tratto spunto dalle danze rituali per costituire a partire da quel linguaggio nuove performance, costruendo maschere innovative e ideando personaggi diversi, liberamente ispirati ai tipi già conosciuti e utilizzati comunemente a Bali.

Negli ultimi venti anni sempre più donne hanno cominciato ad avere uno spazio più significativo anche in forme di spettacolo solitamente considerate appannaggio degli uomini, come nel caso del topeng, una delle danze mascherate maschili per eccellenza. Nel dramma mascherato del topeng, cinque danzatori eseguono coreografie usando diverse maschere: solitamente questa rappresentazione avviene in un contesto rituale, mentre quando la danza viene mostrata a scopo di intrattenimento, solo una maschera è utilizzata. ${ }^{3}$ Le storie su cui si basa il topeng sono le cronache babad sui leggendari re balinesi. Questa danza viene eseguita dagli uomini, per rispecchiare esattamente il genere di tutti i personaggi rappresentati, ma anche alcune donne hanno iniziato a danzare e impersonare le storie del topeng.

Anche questo fenomeno è un sintomo di una profonda innovazione rispetto ai canoni delle rappresentazioni tradizionali. All'interno del complesso delle arti performative balinesi, gli uomini hanno sempre ricoperto incarichi di maggior rilievo nel contesto

\footnotetext{
${ }^{3}$ Coldiron M., Palermo C., Strawson T., 2015, pp. 464-492.
} 
religioso, oltre ad essere la maggioranza dei danzatori. Tradizionalmente alle donne balinesi è riservato un periodo di formazione artistica nel breve periodo della infanzia o della adolescenza, ma difficilmente, una volta raggiunta l'età adulta, si continua a mantenere una qualche forma di training artistico o un ruolo sociale funzionale alle rappresentazioni. Non viene concesso molto tempo per esercitarsi nelle arti della danza, perché il ruolo religioso della donna si concentra maggiormente sull'assolvere alla preparazione delle offerte quotidiane e nella gestione della casa e della famiglia.

Le danze templari e quelle organizzate negli hotel o a scopo di intrattenimento turistico sono eseguite da giovani compagnie di danzatori, che includono a volte anche delle ragazze, mentre l'unica danza che prevede la presenza di donne più mature già sposate è nella processione rituale del mendet in cui viene tradizionalmente accolta o congedata la presenza degli dei in occasione delle festività più importanti dei templi del villaggio.

Con una maggiore apertura al mondo occidentale nel secolo scorso, le accademie artistiche balinesi hanno avvicinato e formato professionalmente anche un discreto numero di studenti stranieri. Emblematica è la figura di Rucina Ballinger, nota per essere l'unica donna ad avere potuto impersonare Rangda. Ballinger arrivò a Bali nei primi anni '70 per studiare danza e si stabilì poi definitivamente nell'isola, diventando una figura fondamentale per la conoscenza all'estero delle arti performative balinesi e per gli scambi culturali tra studiosi occidentali e autoctoni. Rangda è una delle figure spiritualmente più potenti e distruttive che esistano nel patrimonio mitologico e religioso balinese, venendo di solito interpretata dagli uomini perché ritenuti fisicamente e spiritualmente più forti, in grado di sopportare meglio il potere contenuto dalla maschera.

Più la danza è legata al contesto rituale e religioso, maggiore è il rischio di travisare il suo significato profondo; esportare e rendere comprensibili forme di rappresentazione come il sacro Calonarang al di fuori del proprio specifico contesto templare risulta molto complesso. Riprendere solo l'aspetto tecnico del dramma rituale e replicarlo in presenza di spettatori occidentali o senza che questi abbiano la minima nozione del significato del dramma, può rivelarsi una operazione che snatura il senso della rappresentazione riducendola ad una visione esotica che non racchiude più in sé i presupposti per cui si è sviluppata. Secondo la visione di Strawson, in questo processo la performance che prevede l'uso della maschera ha un vantaggio nel passaggio da un contesto tradizionale ad uno totalmente estraneo, perché mantiene la sua efficacia espressiva anche nei confronti di un pubblico che non è a conoscenza dei riferimenti mitologici o narrativi. 
Questo è dovuto al fatto che gli elementi comuni delle maschere sono archetipici e agiscono ad un livello maggiormente immediato e riconoscibile rispetto al linguaggio astrattamente codificato della danza: questa immediatezza espressiva è in grado di superare le barriere del linguaggio parlato, della difficile interpretazione delle strutture sonore e della codifica del significato mitologico e culturale a cui si fa riferimento. ${ }^{4}$

Secondo Coldiron, in ogni performance a Bali, sia in quelle templari che turistiche, $\mathrm{i}$ danzatori non cambiano necessariamente approccio, ma sanno mantenere in ogni occasione il giusto equilibrio tra il puro aspetto dell'intrattenimento e la consapevolezza o il rispetto nel portare avanti una tradizione sacra. Già a partire dalle grandi epiche del Rāmāyana e del Mahābhāratha, sono indicati espliciti riferimenti a danze tradizionalmente performate dalle donne, così come nel Nātyaśāstra, il trattato sul teatro/danza indiano, viene sottolineato il ruolo femminile nella pratica performativa, questo parallelamente al periodo storico in cui in occidente, ad esempio nel teatro greco, la figura della donna non aveva nessun ruolo attivo nel lavoro attoriale. ${ }^{5}$ La questione più rilevante ai fini dell'analisi proposta riguarda l'interrogativo di una presenza prevalentemente maschile nella rappresentazione della figura di Rangda, che trae le sue origini dalla tradizione delle dee terrifiche femminili del retaggio religioso hindu. È la performance di un uomo a rendere artisticamente presentabile la figura di Rangda, a sostenere la sua potenza. Anche se la danza a Bali non è appannaggio esclusivamente maschile, le danze che predispongono nella loro natura una possessione violenta o che hanno a che fare con fattori sovrannaturali di difficile controllo vengono normalmente assegnate agli attori più anziani ed esperti, che sono in grado di gestire la potenza magica derivata senza venirne così sopraffatti.

\footnotetext{
${ }^{4}$ Ivi, p. 480.

${ }^{5}$ Madhavan A., 2015, pp. 345-355.
} 


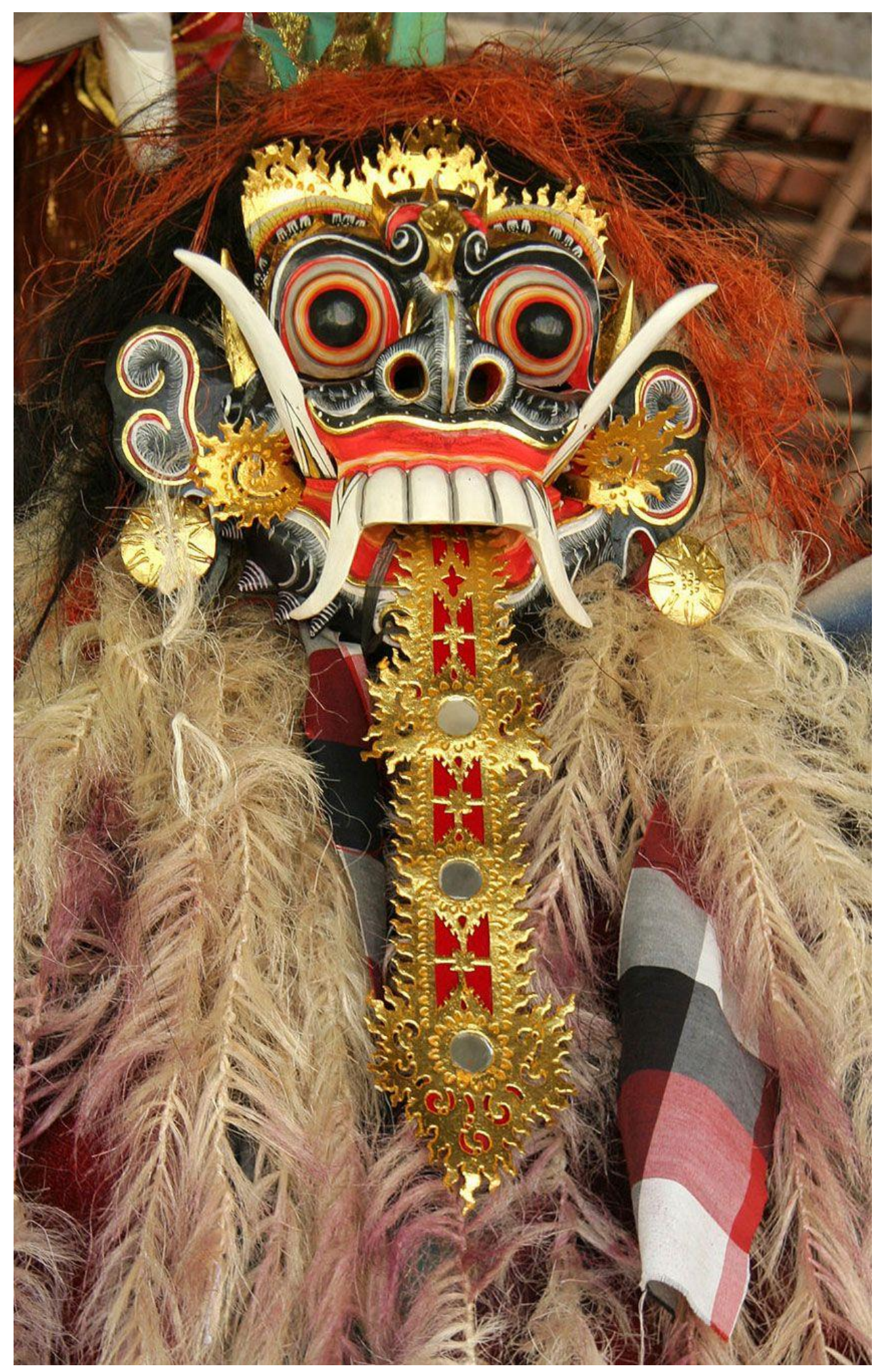




\section{Adattamento culturale del teatro balinese}

Tra le istituzioni accademiche che hanno un ruolo attivo nella formazione professionale dei performer c'è l'Indonesian University of Arts, conosciuto anche come College of Arts di Denpasar, fondato nel 1967. Da un lato le accademie artistiche hanno garantito che alcune forme di danza venissero preservate e tramandate. D'altro canto, tra le critiche che vengono mosse alla scolarizzazione delle numerose forme di teatro danza, c'è la responsabilità di aver appiattito le peculiarità degli stili variabili regionali o dei villaggi, in un processo di standardizzazione delle forme artistiche che ha portato alla progressiva scomparsa di tradizioni familiari o di piccole realtà con le loro usanze caratteristiche. ${ }^{6}$ Per alcune danze di natura secolare, come ad esempio il legong, la transizione da forma di intrattenimento nell'ambiente della corte per le più alte caste, a spettacolo turistico, ha costituito un naturale processo di adattamento avvenuto al di fuori del contesto sacrale.

La differenza rispetto al passato è consistita in un progressivo accorpamento delle danze tradizionali, che ora nelle performance turistiche vengono rappresentate come un insieme unico, utilizzando come accompagnamento musicale uno stile di gamelan ancora più ritmico e aggressivo, il gong keybar gamelan, affermatosi nel corso dei primi decenni del ventesimo secolo. La generale tendenza delle performance turistiche porta ad una condensazione di tutti gli avvenimenti narrativi e delle sezioni di danza che devono essere eseguite in una forma ristretta, in una dimensione spettacolare più fruibile ad un pubblico non esperto. Le danze sono più brevi, performance come il Barong, costituite per durare una intera notte, si svolgono nell'arco di un'ora, e danze come il legong vengono presentate insieme ad un mix di altre musiche in brevi spettacoli della durata di qualche minuto, mentre solitamente veniva dedicato al solo legong anche un'ora di esecuzione. Le musiche che in passato erano associate ai tipi di danza, mantenendo differenti intonazioni e usi di scale specifiche e varie per gli strumenti, non vengono sempre eseguite rispettando i criteri estetici a cui rispondevano, senza applicare come un tempo una più chiara distinzione tra sonorità più aspre (bobot), o più dolci (manis). È anche vero che l'avvento della tecnologia e delle registrazioni ha permesso la diffusione di performance di danza, specialmente tratte dai festival di maggiore importanza a Bali nei principali villaggi, come il Bali Arts Festival. Alcuni gruppi di performers sono riusciti a registrare antiche versioni delle danze tradizionali rintracciando i maestri che operavano

\footnotetext{
${ }^{6}$ Davies S, 2006, pp. 314-341.
} 
in passato, come è accaduto con le testimonianze della tradizione del legong raccolte nella zona del villaggio di Peliatan, da parte del gruppo di danza Dharma Purwa Jati.

È notevole lo sforzo di alcune istituzioni per rivitalizzare e reintegrare nella formazione e nella pratica alcuni generi di performance in via di estinzione, come ad esempio lo stile gambuh, o la danza arja. I più significativi cambiamenti sociali e culturali sono indubbiamente correlati al destino delle arti performative e al loro mantenimento o declino. Le celebrazioni tradizionali che si svolgono nell'ambito templare e nelle realtà dei villaggi spesso non procurano sufficiente introito economico che permetta agli artisti di occuparsi esclusivamente del loro ambito, rendendolo una professione autonoma. Il mercato dell'intrattenimento turistico ha un ruolo fondamentale nell'organizzazione del lavoro quotidiano degli artisti professionisti, che riescono a mantenere un livello tecnico generalmente più alto.

Di conseguenza anche il settore del turismo opera un importante discrimine per la sopravvivenza o il declino di una determinata tradizione artistica, assicurando in base alla fruibilità il suo successo. In alcuni casi i proventi delle performance turistiche possono aiutare a ristabilire e riportare alla luce alcune forme di danza tradizionale, garantendo un apporto di lavoro costante per i gruppi di danzatori e permettendo di ridistribuire i fondi per la comunità o per le cerimonie templari. Uno dei rischi più concreti della commercializzazione delle danze balinesi risiede nella possibilità che esse vengano riproposte in una versione estremamente standardizzata e professionalizzata, perdendo il loro intrinseco legame con la dimensione religiosa e con le peculiarità delle rappresentazioni locali. Le varianti regionali nell'utilizzo degli accessori o di tecniche codificate, o le importanti differenze che sussistono a livello narrativo, che rendono il patrimonio mitologico un insieme variegato di versioni della stessa matrice originaria, rischiano di perdersi in una omologazione accademica che uniforma verso uno stile dominante tutte le performance. L'identificazione che la comunità balinese può recepire dalle rappresentazioni dei gruppi locali, ad esempio i sekan Barong nei villaggi, può perdersi, e rimanere un accessorio vuoto privo di un legame con le varianti personalizzate. Alcune forme di spettacolo standardizzato non rispecchiano l'identità di tutte le comunità balinesi da cui provengono, e non suscitano il medesimo coinvolgimento che permette a queste di collegarsi alla funzione per cui inizialmente erano state ideate.

Picard rileva una certa capacità dei balinesi di essere riusciti a mantenere il proprio retaggio culturale intatto senza sacrificare i propri valori in cambio del profitto 
monetario. ${ }^{7}$ Fin dagli anni '70, a partire dagli studi dell'antropologo americano Philip McKean, l'idea risultante della reazione dei balinesi all'avvento del turismo di massa è che essi abbiano saputo cogliere le opportunità economiche intrinseche al fenomeno senza risultare da questo processo snaturati o alla deriva rispetto alla propria identità culturale. Anzi, per McKean sarebbe proprio il rinnovato benessere economico a poter garantire di preservare alcune tra le tradizioni di teatro/danza più a rischio.

Nella concezione di cultura come performance, proposta da Milton Singer, le manifestazioni artistiche balinesi rientrerebbero in questo campo, con la differenza che, mentre quelle sacre sarebbero predisposte per una platea "divina" allo scopo, oltre che di protezione e di sacrificio, di salvaguardare i valori propri della comunità, le performance turistiche, non contravvenendo affatto al proposito di quelle sacre, potrebbero fornire un maggiore apporto economico permettendo così la sopravvivenza di quei valori da preservare e coltivare. I due mondi possono convivere così in mutuo accordo, da una parte l'assetto religioso e tradizionale dei festival templari garantisce che gli attori non perdano il loro spirito di autenticità, dall'altra le performance commerciali portano benessere e permettono una rinnovata prosperità. La reazione di base dei custodi della tradizione balinese non è stata di annullamento o sostituzione della propria identità culturale, essi hanno aggiunto nuove forme di intrattenimento senza abbandonare quelle preesistenti. Per una serie di motivazioni sociali e religiose, l'aspetto secolare e quello sacro rimangono collegati in termini di struttura, ma fondamentalmente separati dal punto di vista dei contenuti. ${ }^{8}$

La formula sviluppata dal governo balinese per occuparsi del settore si è strutturata nell'idea di un "turismo culturale" (pariwisata budaya), che dalla seconda metà del '900 ha coinvolto le maggiori agenzie per la promozione del turismo, dell'intrattenimento e della cultura. Indubbiamente il patrimonio artistico e religioso rendeva Bali tra le mete più conosciute al mondo, ma l'invasione turistica di stranieri visitatori avrebbe potuto rappresentare un problema, considerando il fenomeno come una sorta di "inquinamento" culturale: c'era il timore che Bali si sarebbe uniformata progressivamente ad altre realtà, perdendo parte della propria identità. Il turismo culturale rappresentò una soluzione a lungo termine per occuparsi di entrambi gli aspetti, da un lato per favorire lo sviluppo

\footnotetext{
${ }^{7}$ Picard M., 1990, pp. 37-74.

${ }^{8}$ Mc Kean F,1973, p. 1.
} 
economico del settore turistico, dall'altro per valorizzare e preservare le tradizioni artistiche locali. La questione fondamentale che pone Picard è cosa sia realmente accaduto alle performance artistiche una volta che esse siano state messe al servizio dell'intrattenimento turistico. La visione manichea di un "inquinamento culturale" piuttosto che di un utopico "rinascimento" delle arti a seguito dell'interesse dei visitatori non fornisce un quadro accurato e realistico della situazione.

Anche Bruner si è interrogato sull'individuare le differenze specifiche tra le espressioni culturali autentiche pre-esistenti all'avvento del turismo di massa, rispetto alle forme di intrattenimento nate e sviluppate specificatamente per i non balinesi. ${ }^{9}$ Il turismo internazionale è arrivato a Bali anche allo scopo di conoscere la cultura balinese, e la popolazione locale ha dunque dovuto creare una adeguata rappresentazione delle proprie tradizioni, basandosi sul proprio retaggio ma anche sulle aspettative - presunte, o previste - dei visitatori. Le forme di teatro/danza rinnovate e conosciute in tutto il mondo come simbolo di Bali hanno costituito per la cultura balinese stessa uno specchio in cui riflettere sulla propria rappresentazione, riconsiderando i propri valori.

La concezione stessa di "arte" è una distorsione data da una visione esterna, dal momento che si tratta più di un servizio e di una occupazione della comunità nei confronti degli dei e della sua stessa sopravvivenza. Coloro che si occupano di arte eseguono qualcosa di altamente specializzato, secondo l'uso del prefisso juru («specialista») applicato ai vari ambiti della danza o della musica. Gli aspetti coreografici e drammaturgici non sono differenziati, ma coesistono ad un livello profondo. Naturalmente la danza e il dramma non sono il ritratto immobile di una rappresentazione, ma è nell'azione drammatica che si manifesta l'aspetto rituale, non potendo creare una cesura tra l'intrattenimento e il rito religioso. ${ }^{10}$

Il contesto tradizionale in cui si svolgono le performance drammatiche è all'interno delle celebrazioni templari, o in occasione di cerimonie religiose come nei riti di passaggio, nelle cerimonie funebri o in pratiche di esorcismo. Anche le danze eseguite nel contesto della corte, per i Raja, avevano lo scopo rituale di glorificare il potere regale, di derivazione divina, e di onorare gli antenati di stirpe nobile. Con la diminuzione del potere dei sovrani, specialmente a seguito dell'occupazione olandese, le corti non furono più in grado di sostenere le antiche forme di intrattenimento e di rappresentazione teatrale

\footnotetext{
${ }^{9}$ Bruner E.M., 1986, p. 28.

${ }^{10}$ Belo J., 1960, p. 115.
} 
come facevano un tempo, e il patrimonio di tali generi di danza passò alle comunità dei singoli villaggi, dove lo stile e le coreografie subirono un processo di rinnovamento e di modifica, adattandosi alla nuova situazione.

Il successivo sviluppo del turismo negli anni '60 arrivò dopo un periodo di difficoltà politica ed economica a seguito delle spinte indipendentiste e dei conflitti mondiali. Il governo ricominciò ad interessarsi alla salvaguardia delle arti performative balinesi e il rinnovato afflusso turistico permise al settore di risollevarsi ed espandersi. Nacquero così numerose istituzioni per diffondere e preservare il patrimonio culturale ed artistico, prendendo da un certo punto di vista il posto delle antiche corti nel creare situazioni e luoghi di incontro e scambio tra maestri e allievi, nel favorire la creazione di nuovi stili di danza e musica e nel mantenere intatte le tradizioni, finanziando e sostenendo sempre più progetti artistici.

Le performance specificatamente pensate e costruite per intrattenere il pubblico turistico in visita a Bali si sono sviluppate solitamente nei paesi più conosciuti e popolari, inseriti nel giro dei tour per le vacanze, come Batubulan o Peliatan, o negli hotel internazionali e nei resort di Ubud e Sanur. I gruppi di artisti che vengono assunti per le performance turistiche sono gli stessi che si esibiscono per il pubblico di balinesi nelle cerimonie templari ufficiali. Vi è una certa difficoltà ad accedere direttamente al mercato turistico, per una troupe di danzatori o musicisti emergenti, perché gli ingaggi sono gestiti da agenzie intermediarie, che dettano condizioni contrattuali specifiche e in alcuni casi approfittano della competizione tra gli artisti per ottenere maggiore guadagno, o per avere un controllo sulle modalità e il contenuto degli spettacoli proposti. Degli introiti che arrivano direttamente alla troupe di danzatori, una parte viene solitamente reinvestita per il mantenimento o il miglioramento di costumi e accessori di scena, o dedicata come offerta alle celebrazioni annuali, oltre al normale pagamento delle tasse governative e della percentuale dovuta alle agenzie.

Per mantenere un determinato standard sulle performance, le autorità governative hanno stabilito che per lavorare nel mercato turistico sia necessaria una speciale licenza (Pramana Patram Budaya) rilasciata dal Concilio delle Arti. Rispetto alla grande diversità che caratterizza le numerose danze balinesi, queste si svolgono quasi sempre su setting già prestabiliti, ad esempio con scenografie che ricordano l'entrata di un tempio, montato su un palco davanti ad una platea di posti a sedere. Per i tipi di spettacolo che già appartenevano a generi di intrattenimento, il passaggio da un pubblico autoctono ad uno 
più eterogeneo non ha sostanzialmente modificato l'intento originario, variando verso una progressiva semplificazione dei contenuti.

Uno dei motivi di grande fascino che attrae il pubblico è invece costituito proprio da quel tipo di performance, come nel caso del Barong, che prevede un rituale di possessione, in cui la trance dei danzatori viene messa in scena in maniera particolarmente spettacolarizzata.

Il Barong deve la sua estrema popolarità all'interesse per le maschere di Rangda e Barong ${ }^{11}$, e la descrizione che più spesso viene utilizzata è che la "strega" e il "drago" (immagini che sono liberamente tratte dall'immaginario mitologico fiabesco occidentale, più vicine rispetto all'originale iconografia della religione hindu), siano i rappresentanti delle forze del bene e del male, e si avvicendino in un continuo conflitto. Questa interpretazione non appartiene alla visione balinese, in cui ciò che viene evidenziato è piuttosto un tentativo di stabilire un contatto con forme di potere sovrannaturale, secondo i principi della cultura hindu e della concezione magica del tantrismo, per potersi confrontare con la dimensione delle forze più distruttive della realtà. Attraverso le dovute procedure rituali è possibile incanalare in modo costruttivo l'intervento di Rangda e Barong, la loro natura ambivalente, poiché entrambe le figure hanno una componente creativa e insieme distruttiva.

Il rituale sacro che mette in scena il confronto a partire dalle leggende dell'antico regno di Giava è il Calonarang, che racchiude in sé le caratteristiche di una performance e di un esorcismo, il cui scopo è contenere il potere distruttivo delle forze sovrannaturali evocate e messe in scena con le maschere tradizionali. Il luogo e la situazione temporale in cui si svolge il rituale devono essere propizi, e perché il rito svolga la sua funzione apotropaica occorre che l'attore che interpreta Rangda sia in grado di accogliere e sperimentare la possessione divina dello spirito, una manifestazione della dea Durga, per poter garantire la protezione della comunità dalle forze del male e della distruzione. La parte della performance che ha costituito l'attrattiva più forte anche per i visitatori stranieri consiste nell'attacco del seguito del Barong, in cui i guerrieri posseduti e in preda alla trance, rivolgono su di sé i pugnali rituali (kriss), soggiogati dal potere di Rangda, uscendone illesi. ${ }^{12}$

\footnotetext{
11 Picard M., cit., p. 56.

12 Per maggiori dettagli, vedi Eiseman, 1992, $146-160$.
} 
Una delle performance di Calonarang di cui si ha notizia ufficiale fu rappresentata nel 1931 in occasione della esibizione coloniale di Parigi, in cui danzatori da Singapadu eseguirono un breve estratto del dramma utilizzando maschere non consacrate ritualmente. I primi studi sulla trance e sullo spettacolo balinese furono compiuti nello stesso periodo storico dagli antropologi Jane Belo, Margaret Mead e Gregory Bateson, che documentarono per anni le attività dei gruppi teatrali del Barong nei villaggi più attivi culturalmente, specialmente nella zona di Batubulan. ${ }^{13}$ Per documentare il fenomeno, vennero organizzate anche alcune rappresentazioni durante il giorno, che vennero fotografate e filmate con l'intervento del pittore e musicista tedesco Walter Spies. Stimolati dal successo e dall'interesse accademico suscitato dal Calonarang, alcuni gruppi di danzatori e musicisti iniziarono ad organizzare e far conoscere il dramma a scopo di intrattenimento, sulla base strutturale e narrativa del rituale. Furono effettuate alcune modifiche sostanziali, riducendo di molto la durata della performance e schematizzando l'impianto narrativo, e venne di fatto riallestito un nuovo spettacolo arrangiato per essere alla portata di turisti stranieri e chiamato più semplicemente "Barong e la danza del kriss".

Dopo una interruzione del flusso di visitatori nel periodo della Seconda guerra mondiale, i gruppi del Barong, specialmente tra i villaggi di Batubulan e Singapadu, ricominciarono negli anni '60 a riproporre la nuova versione semplificata del dramma sacro ai turisti sempre in maggiore afflusso, riuscendo a creare un vero e proprio business commerciale basato sul Barong, in cui anche più gruppi di danzatori eseguivano brevi spettacoli, ogni giorno, della durata di un'ora circa.

Anche altre danze sacre che prevedono la trance sono diventate molto popolari e sono state riadattate e pubblicizzate per il settore turistico, divenendo il vero e proprio simbolo caratteristico della cultura balinese. La danza sanghyang, praticata in origine a fini di esorcismo, è stata modificata come genere di intrattenimento spettacolare, sia nel caso del sanghyang jaran, in cui il performer, solitamente un giovane accompagnato da un coro maschile (cak), viene posseduto dallo spirito di un animale e imita le sue movenze, e anche per il sanghyang dedari. ${ }^{14}$ Alcuni tra questi tipi di danza sono stati modificati e uniti insieme in un'unica breve rappresentazione, mescolando diversi stili di musica e di

\footnotetext{
13 Picard M., cit., p. 58.

${ }^{14}$ Per una descrizione del sanghyang dedari, vedi Eiseman F., cit., p. 135.
} 
coreografia. Ad esempio, la creazione del kecak proviene dal coro cak presente nel sanghyang, riadattato inserendo i movimenti dello spettacolo del baris.

Fin dai primi anni '20 del Novecento, i gruppi di danzatori che frequentavano gli artisti e i visitatori occidentali vicini a Walter Spies idearono un nuovo spettacolo basato sulle vicende narrate nel Rāmāyana, accompagnate dal coro cak e dall'orchestra gamelan. Lo spettacolo fu una vera e propria rivisitazione artistica che riscosse molto successo tra il pubblico di occidentali, divenendo conosciuto tra i turisti come "Danza della Scimmia", in riferimento al ruolo degli attori che interpretano l'armata di scimmie per salvare la principessa Sita, la sposa di Rāma. Questa performance nel corso di un secolo ha continuato a rinnovarsi, con l'apporto di danzatori professionisti formati nelle accademie indonesiane, talvolta reintroducendo elementi caduti in disuso dalle antiche danze tradizionali del sanghyang. Alcuni membri della comunità balinese si sono espressi molto negativamente sull'uso improprio delle danze rituali, che venendo presentate come performance di intrattenimento vedrebbero snaturata la loro funzione e importanza.

Ci sono molte interferenze e sovrapposizioni tra i retaggi della tradizione balinesi, che si sono costituite e adattate nel corso di secoli di storia, e tra le rappresentazioni che appartengono alla storia recente, che risentono degli influssi dello sviluppo del mondo contemporaneo, in alcuni casi espressamente ideate per rispondere ad una esigenza di intrattenimento turistico, senza per questo rimanere slegate dal contesto religioso e culturale. Nel caso della danza del Barong, insieme al resto degli spettacoli che traggono libero spunto dai rituali sacri di esorcismo e di possessione, queste forme di teatro danza si sono evolute in modo diverso e continuano tutt'ora ad ampliare il proprio significato. In qualunque situazione siano rappresentate, le figure di Rangda e del Barong evocano un legame con le forze sovrannaturali di cui sono simbolo, non potendo totalmente essere separate dai loro scopi rituali e dal significato che rivestono nella mitologia balinese. La relazione tra la dimensione rituale e quella spettacolare è fluida, e può portare a nuove idee di intrattenimento in cui i significati narrativi originari non sempre sono stati mantenuti nella loro forma originaria, pur utilizzandone i contenuti formali. In diverso modo, anche senza il contesto rituale, una rielaborazione anche profonda delle forme di teatro danza tradizionale porta ad un continuo interrogativo sugli elementi stessi della cultura, invitando ad una autoriflessione sui valori e le strutture su cui si reggono. 


\section{Sulle danze sacre e profane}

Le autorità religiose e governative balinesi hanno operato in modo deciso per evitare che la confusione tra l'aspetto rituale e l'intrattenimento portasse a forme di commercializzazione eccessiva e di profanazione dell'aspetto sacro delle rappresentazioni. Dopo essere stato fonte di aspro dibattito culturale e politico, il turismo continua comunque ad essere percepito come una risorsa preziosa che possa portare anche ad uno sviluppo per l'ambito artistico, piuttosto che come una minaccia per i valori religiosi tradizionali. Uno dei momenti cruciali in cui si è dibattuto su quali aspetti fosse opportuno preservare, è rappresentato dal Seminario sulle danze sacre e profane (Seminar seni sacral dan provan bidang tari $)^{15}$, che si è tenuto nel marzo del 1971 a Denpasar, ad opera dell'Ufficio balinese del Dipartimento dell'Educazione e della Cultura (Kanwil Departemen P dan K Propinsi Bali).

Nel corso del seminario l'obbiettivo principale è stato quello di fornire indicazioni precise alle principali organizzazioni che si occupavano di turismo, per costituire insieme una linea comune sul patrimonio delle danze sacre rispetto ad un tipo di arte considerato "secolare", profano. Una distinzione tra i due generi avrebbe così permesso la salvaguardia della tradizione religiosa contribuendo ad uno sviluppo commerciale adeguato al settore dell'intrattenimento turistico. Furono invitati numerosi esponenti dell'ambiente religioso e artistico/culturale balinese per esprimersi sulla questione e nel corso dell'evento è emersa la reale portata della problematica. In riferimento all'arte balinese, la stessa terminologia della cultura e della religione non permette di articolare una chiara opposizione tra i due concetti di arte sacra e profana. Non è stato possibile separare le due realtà, dal momento che tutto il complesso è costituito sulle medesime basi culturali, che uniscono insieme la dimensione divina e l'intrattenimento fine a se stesso. Una distinzione più vicina al vissuto della cultura balinese poteva essere costituita dal confronto tra agama e adat, che rispettivamente hanno il significato di "religione" e "tradizione". Anche questa differenziazione semantica appare fumosa e non risolve del tutto il problema, dal momento che nel campo del termine adat rientra anche la percezione di un immutabile ordine sociale che ha derivazione universale e divina, poiché non c'è un solo aspetto della cultura balinese che non sia intrecciato con un substrato religioso. Utilizzando ugualmente questa opposizione terminologica, si è potuta delineare una

\footnotetext{
${ }^{15}$ Poyek Pemeliharaan dan Kebudayaan Daerah, 1971.
} 
sommaria differenza tra sacro e profano. Si è dunque definito che ciò che riguardasse l'aspetto agama costituisse la base irrinunciabile dell'identità culturale balinese.

Una delle strategie che sono state messe in atto per evitare di commerciare illegittimamente contenuti a sfondo religioso, è derivata da una maggiore tendenza a separare concettualmente ciò che riguarda più strettamente la religione, nel senso dell'insieme delle norme rituali prescrittive tramandate dai sacerdoti, o le procedure di purificazione e l'osservazione dei sacrifici e delle offerte templari e familiari, da tutto quello che invece rientra nella più ampia concezione di mondo sovrannaturale, regolato dalle forze della śakti, l'energia divina. Nell'interpretare e divulgare ad un pubblico di stranieri i principi fondativi dei contenuti del teatro balinese, viene privilegiata la visione di un conflitto tra due opposizioni morali del bene (kebaikan) e del male (keburukan). Il confronto tra Barong e Rangda, tuttavia, non può essere spiegato solo in termini di un giudizio morale unidirezionale, dal momento che definirlo in modo univoco come scontro significa commettere una inesattezza di concetto.

I festival musicali a Bali sono principalmente di carattere sacrale. ${ }^{16}$ La maggior parte degli eventi si svolge al calare della notte, e gli spettatori del villaggio indossano costumi tradizionali. Solitamente per le rappresentazioni che si svolgono nella zona esterna al tempio è possibile anche scattare fotografie o fare alcune registrazioni, mantenendo un comportamento decoroso e un abbigliamento coperto, con la donazione di una piccola offerta. Il Barong che negli ultimi anni viene presentato ai turisti è uno spettacolo che racchiude frammenti di danze tradizionali balinesi, che raccontano una vicenda di facile comprensione, tratta dal poema del Mahabharata. Le maschere ed i costumi che vengono utilizzati non sono sacri. Il Barong così rappresentato è sempre un barong ket, con la presenza del leone mitologico, che manifesta più spiccatamente un carattere più umoristico e piacevole. Si inizia sempre con una parte solistica con il Barong, a cui seguono alcuni frammenti di danze balinesi tradizionali, solitamente con due giovani danzatrici seguite da danzatori travestiti da scimmie. Compare infine Rangda, che spinge i seguaci del Barong con i suoi poteri magici a ferirsi autonomamente, facendoli cadere in uno stato di trance, che nello show turistico è nella maggior parte dei casi simulata. L'intera performance è accompagnata da un'orchestra gamelan. Il carattere fondamentale di questa danza è la sua natura di puro intrattenimento turistico e rimane quindi una versione semplificata del dramma.

\footnotetext{
${ }^{16}$ Eiseman F., cit., p. 281.
} 
Il gruppo di danze denominate come bebali rientra nel genere di performance associato alle celebrazioni templari più importanti, ma a differenza delle danze più sacre, wali, mantengono un legame meno forte con la dimensione del sacro. Questo genere contiene quasi sempre degli elementi narrativi, che possono favorire l'attenzione e la partecipazione delle divinità che assistono, ma che non sono nate allo specifico scopo rituale. Il fine religioso è comunque ben chiaro a tutti i partecipanti e gli spettatori, che vengono intrattenuti come un tempo era riservato ai membri delle caste più alte, per far trascorrere le lunghe giornate di festeggiamenti templari. ${ }^{17}$ Un esempio di danza wali è il rejang, eseguita dal gruppo delle giovani donne del villaggio, non professioniste, che utilizzano movimenti semplici ma di grande espressività. Le danze si svolgono durante il giorno, al pomeriggio, fin dentro il jeroan, la parte più interna del tempio, dove gli spettatori principali sono gli dei. La danza complementare al rejang è il baris gedè, eseguita da un gruppo di uomini in occasione degli odalan. Il termine baris si riferisce alla formazione pseudo-marziale e militaresca replicata dai danzatori, che procedono come dei soldati indossando alcune armi cerimoniali, accompagnati dal suono dei gong.

La tradizione del genere di danze considerate oggi come bebali è collegata alla cultura di origine Giavanese di matrice induistica, importata nei secoli dalle tradizioni del regno di Giava e dalle influenze della cultura artistica e spirituale indiana. Nel corso del XIV secolo, Bali venne annessa ufficialmente ai possedimenti dell'Impero Majapahit, che avrebbe mantenuto la propria supremazia politica sul territorio fino alla sua caduta provocata dal successivo avvento dell'Islam. Le danze bebali rappresentano il retaggio della cultura di corte importata e sviluppata durante il periodo Majapahit, in cui le danze più sofisticate ed eleganti erano celebrate a scopo di intrattenimento nei palazzi dei sovrani, in presenza dei più alti funzionari di corte e delle caste più alte. I sovrani hindu e il loro seguito di consorti e consiglieri sostennero economicamente e politicamente lo sviluppo delle arti a corte, talvolta anche esibendosi come danzatori dilettanti e prendendo parte all'intrattenimento durante le celebrazioni più importanti. Danzatori professionisti erano mantenuti a corte e si specializzarono per offrire spettacoli di sempre maggior pregio artistico e raffinatezza. Alcune delle danze che ricalcano questo stile sono il gambuh, i cui temi narrativi vertono sulla vita di corte, prendendo come fonte il poema Malat, che pone al centro dei suoi racconti le avventure e le vicissitudini del celebre principe Panji Inu Kertapati, e la sua promessa sposa Candra Kirana. Le vicende sono

\footnotetext{
${ }^{17}$ I Made B. - De Boer Frederik E, 1981, p. 26.
} 
narrate nella antica lingua kawi, il linguaggio che veniva utilizzato nelle corti di Bali e Giava nel periodo medievale.

È a seguito del seminario del ' 71 che è emersa la distinzione più netta tra le categorie di danza wali, bebali e balih-balihan. Le danze wali solitamente sono mancanti di un contenuto narrativo, come accade nel pendet, nel rejang, il baris gede e nel sanghyang. Le danze cerimoniali, chiamate bebali, vengono eseguite nel cortile centrale del tempio (jaba tengah), come accompagnamento ai veri e propri rituali, includendo anche elementi narrativi, come accade nel caso del topeng o del gambuh.

Le danze balih-balihan non sono consacrate, e vengono eseguite nel cortile esterno del tempio (jaban), o anche in altre situazioni come forma di intrattenimento puro. Da questa differenziazione sommaria, frutto dell'accordo e della partecipazione di intellettuali e personalità religiose della società balinese, emerge l'opinione che nell'ultima categoria non ci sia un contenuto esattamente prestabilito, ma che possano esservi inseriti tutti quei generi di performance che non rientrano specificatamente nei tipi di danza segnalati precedentemente. Il termine balih- indica precisamente un atto che nasce per essere "guardato", ed è quindi traducibile con il termine "spettacolo"; tuttavia, la distinzione tra le prime due classificazioni dei generi è più complessa da gestire. Bebali è un termine di derivazione sanscrita che può essere tradotto come "offerta", mentre wali significa "cerimonia". Questa distinzione intellettuale in realtà trova una certa difficoltà ad essere applicata nella pratica cerimoniale, perché non sempre è possibile validare i criteri di categorizzazione. Ciò che caratterizza le danze wali è la loro natura intrinseca di offerta divina: nel contesto di una celebrazione religiosa, queste sono parte integrante e naturale del rito, eseguite per esclusiva presenza degli dei. La difficoltà maggiore viene riscontrata quando si ritrova anche questa idea di fondo nella celebrazione delle danze bebali. La classificazione del '71 è stata attribuita in termini di contenuto, anche se gli stessi danzatori balinesi sono rimasti nel tempo in disaccordo su quali danze fossero da inscrivere in una determinata categoria.

L’Ufficio del Dipartimento di religione si è occupato di rivedere la iniziale nomenclatura in un altro seminario nel 1978, focalizzando maggiormente l'attenzione sulla funzione delle danze, rispetto ai puri contenuti. Secondo questa direttiva più specifica, è stato possibile far rientrare alcune danze bebali nella categoria wali, nel caso queste fossero inserite contestualmente in una cerimonia templare, o classificandole come balih-balihan se rappresentate come pura forma di intrattenimento. La differenza dunque 
non è assoluta, ma dipende dal contesto e dal significato attribuito di volta in volta dagli officianti, dall'autorità religiosa presente, dal pubblico e dal luogo in cui si svolge la danza. Tutte le danze tradizionali, ovvero quelle che si possono datare prima dell'arrivo del nuovo stile keybar e dello sviluppo turistico dei primi decenni del '900, sono nate e si sono sviluppate in un contesto religioso e rituale. Per la danza del Barong, non c'è stata una precisa indicazione, perché non viene menzionata nella classificazione dei seminari del '71 e del ' $78 .{ }^{18}$ Una ulteriore discriminante è stata applicata nel 1980 dal Dipartimento di Religione stabilendo che per considerare le danze wali gli accessori e gli indumenti dei danzatori dovessero essere consacrati ritualmente, mentre il resto delle performance di intrattenimento, anche se ispirate ai drammi rituali nei contenuti e nell'equipaggiamento, non potessero essere eseguite con elementi religiosi e consacrati. In realtà, nell'osservare come alcune performance del Barong siano organizzate per un pubblico misto di turisti e balinesi, anche uno spettacolo pensato per non essere "ritualizzato" riassume in sé alcuni elementi di carattere religioso, perché è intrinseco nel significato delle maschere e della danza una componente spirituale che non potrà mai del tutto essere eradicata. Senza un'adeguata offerta e un rituale di consacrazione, per alcuni danzatori non è possibile far "vivere" i personaggi, rappresentare Rangda e Barong nella loro potenza, che assicura la riuscita dello spettacolo e ne legittima la sua intera esistenza, assicurando la sopravvivenza di un retaggio tradizionale anche nelle forme di intrattenimento più modernizzate.

Anche gli spettacoli turistici possono avere una rilevanza religiosa, dalla riuscita di questi può arrivare protezione e beneficio, mantenendo un equilibrio tra le forze sovrannaturali che vengono celebrate, e dunque è difficile ritenere che l'aspetto esteriore ed estetico della performance del Barong e Rangda possa essere separabile totalmente dalla sua valenza rituale e dal suo profondo valore spirituale. Contrariamente alle distinzioni espresse dalle autorità politiche o religiose, i performer, danzatori e attori balinesi, continuano a confrontarsi quotidianamente veicolando entrambe le dimensioni del sacro e del profano, muovendosi in un contesto ancora intriso di una profonda spiritualità, in cui il legame con il rito rimane tangibile anche nelle forme di spettacolo più moderne.

\footnotetext{
${ }^{18}$ Picard M., cit, p. 68.
} 


\section{Bibliografia}

AZZARONI Giovanni, Società e Teatro a Bali, Bologna, Clueb, 1994

AZZARONI G., CASARI Matteo, Asia: il teatro che danza, Firenze, La Lettere, 2011

BELO Jane, Trance in Bali, New York, Columbia University Press, 2020 (prima edizione: New York, Columbia University Press, 1960)

BERGER Arthur Asa, Bali Tourism, London, Routledge, 2013

BRUNER Edward M., "Experience and Its Expressions", in Anthropology of Experience, ed. TURNER Victor and Bruner M., Urbana, University of Illinois Press, 1986, p. 28

BÜCHNER Olejsa, Bali: Mass Tourism in Developing Countries, Monaco, GRIN Verlag, 2003

COLDIRON Margaret, PALERMO Carmencita, STRAWSON Tiffany, "Women in Balinese "Topeng": Voices, Reflections, and Interactions", Asian Theatre Journal, Vol. 32, No. 2, 2015, pp. 464-492

DAVIES Stephen, “Balinese Legong: Revival or Decline?”, Asian Theatre Journal, Vol. 23, No. 2, 2006, pp. 314-341

DI BERNARDI Vito, Introduzione allo studio del teatro indonesiano: Giava e Bali, Firenze, Ponte delle Grazie, 1995

DÖRR Jessica, Bali. Country Profile and Tourism, Monaco, GRIN Verlag, 2004

EISEMAN Fred B., Bali - Sekala e Niskala - Essays on Religion, Ritual, and Art, Singapore, Periplus Edition, 2009 (prima edizione: Singapore, Periplus, 1996)

HITCHCOCK Michael, Mr I NYOMAN Darma Putra, I. NYOMAN Darma Putra, Tourism, Development and Terrorism in Bali, Aldershot, Ashgate Publishing Limited, 2012. (prima edizione: Aldershot, Ashgate, 2007).

HITCHCOCK M, AVE J., JAY S.E., GUSTAMI S.P., Indonesian Arts and Crafts, Jakarta, BAB Publishing Indonesia, 2009

HOWE Leo, The Changing World of Bali: Religion, Society and Tourism, New York, Routledge, 2006. (prima edizione: Abingdon, Routledge, 2005)

I MADE Bandem. - DE BOER Frederik E., Balinese Dance in Transition, Kuala Lumpur, Oxford University Press, 1981.

I MADE Bandem., “Barong Dance”, The World of Music, Vol. 18, No. 3, 1976, pp. $45-52$

JOHNSON Henry, "Balinese music, tourism and globalisation: inventing traditions within and across cultures", New Zealand Journal of Asian Studies, Vol. 4, No. 2, 2002. pp. 8-32 University of Otago

MADHAVAN Arya, "Introduction: Women in Asian Theatre: Conceptual, Political, and Aesthetic Paradigms", Asian Theatre Journal, Vol. 32, No. 2, 2015, pp. 345-355

MCCARTHY John, Are Sweet Dreams Made of This? Tourism in Bali and Eastern Indonesia, Northcote (Australia), Indonesia Resources and Information Program, 1994 
MC KEAN Philip F., Cultural Involution: Tourists, Balinese, and the Process of Modernization in an Anthropological Prospective, Ann Arbor (Michigan), University Microfilm International, 1973, p. 1

PICARD Michael, “'Cultural Tourism' in Bali: Cultural Performances as tourist attraction”, Indonesia No. 49, 1990, pp. 37-74, Cornell University Press

PICARD Michael, DARLING Diana, Bali: Cultural Tourism and Touristic Culture, Singapore, Archipelago press, 1996

PICKEL-CHEVALIER Sylvine, Tourism in Bali and the Challenge of Sustainable Development, Newcastle, Cambridge Scholars Publishing, 2018 (prima edizione: Newcastle, Cambridge Scholars, 2017)

POYEK PEMELIHARAAN dan Kebudayaan Daerah, in Seminar Semi Sacral dan Provan Bidang Tari, Denpasar, 1971

SHAVIT David, Bali and the Tourist Industry: a history, 1906-1942, Jefferson (NC), McFarland Incorporated Publishers, 2019. (prima edizione: Jefferson, McFarland, 2003)

VICKERS Adrian, "Bali rebuilds its tourist industry”, Bijdragen tot de Taal-, Landen Volkenkunde, Vol. 167, No. 4 (2011), pp. 459-481

WOOD Robert E., "International Tourism and Cultural Change in Southeast Asia", Economic Development and Cultural Change, Vol. 28, No. 3, 1980, pp. 561-581

YAMASHITA Shinji, Bali and Beyond: Explorations in the Anthropology of Tourism, Oxford, Berghahn Books, 2003

YAMASHITA Shinji, EADES Jeremy S., Globalization in Southeast Asia: Local, National, and Transnational Perspectives, Oxford, Berghahn Books, 2003 